56 cases (37 adult, 19 pediatric) reported to the British Neurological Surveillance Unit (BNSU) and surveyed at the Department of Psychological Medicine, Institute of Psychiatry, London, UK. Male to female ratio was 2:1 for adult cases and 3:2 in children. Approximately two-thirds (57\%) had epilepsy, half the adults were intellectually impaired, and $35 \%$ had a psychiatric disorder. Macrocephaly may have accounted for an unusually high (35\%) incidence of obstetric complications. The obstetric history was not correlated with the complication of epilepsy, low IQ or psychiatric disorder. (Taylor M, David AS. Agenesis of the corpus callosum: a United Kingdom series of 56 cases. I Neurol Neurosurg Psychiatry Jan 1998;64:131134). (Respond: Professor AS David, Department of Psychological Medicine, Institute of Psychiatry, De Crespigny Park, Denmark Hill, London SE5 8AF, UK).

COMMENT. In addition to epilepsy, cognitive impairments and mental retardation, agenesis of the corpus callosum may be complicated by neuropsychiatric disorders, including attention deficit hyperactivity disorder and psychoses. Corpus callosal agenesis is a manifestation of Aicardi and other developmental syndromes. It may also be associated with schizencephaly.

\title{
SCHIZENCEPHALY AND CYTOMEGALOVIRUS INFECTION
}

Two cases of schizencephaly associated with cytomegalovirus (CMV) infection are reported from the University La Sapienza, Rome, and Dibit Scientific Institute, Milan, Italy. In patient 1, MRI showed a left schizencephaly with a polymicrogyric cortex lining the clefts; CMV DNA was demonstrated in the serum on the second day of life. Patient 2 was referred at 4 years of age, and MRI showed a large, open left schizencephaly, surrounded by polymicrogyric cortex, and cerebellar hypoplasia. CMV DNA was detected in the serum, the early history revealed intrauterine growth retardation, microcephaly, petechiae, cataract, hepatospenomegaly,anemia, and periventricular calcifications were present on $\mathrm{CT}$ at 3 months of age. Tests for a genetic origin of the schizencephaly failed to demonstrate the characteristic mutation in the homeobox gene EMX2. (Iannetti P, Nigro G, Spalice A, Faiella A, Boncinelli E. Cytomegalovirus infection and schizencephaly: case reports. Ann Neurol Jan 1998;43:123-127). (Respond: Dr Paola Iannetti, Child Neurology Division, Pediatric Department, University La Sapienza, Viale Regina Elena 324, 00161 Rome, Italy).

COMMENT. Prenatal cytomegalovirus infection may be a factor in the pathogenesis of some cases of schizencephaly and other neuronal migration disorders. Yakovlev and Wadsworth who coined the term schizencephaly (1946) timed the formation of the clefts in the 2 nd month of fetal development.

\section{PAROXYSMAL DISORDERS}

\section{NARCOLEPSY AND CATAPLEXY}

The clinical manifestations and treatment of narcolepsy diagnosed in 51 prepubertal children (29 boys) are reported from the Stanford Sleep Disorder Clinic, Stanford University Medical Center, CA. The mean age was 8 years (range, 2 to 11 ). Diagnosis was based on daytime sleepiness as the initial symptom in 39,10 presented with cataplexy, and 2 had hallucinations and sleep paralysis. In 5 patients who presented at or before 5 years of age, epilepsy was the first diagnosis considered; 3 reported abrupt unexplained falls, clumsiness, day dreaming, night terrors, and intermittent irritability and aggressiveness. The history of daily naps was obtained only after visits to 3 different specialists. Of 46 children older than 5 
years, 36 were referred because of complaints from school: falling asleep in class, day dreaming, abrupt falling, and inattention. Attention deficit disorder was diagnosed in 10, hyperactivity in 12, epilepsy in 23, learning disability in 16 . Behavioral and learning problems prompted examination by school psychologists or counselors in 39 children before the correct diagnosis was established. Cataplexy (sudden loss of muscle tone) was precipitated by laughter, but was accompanied by fear of embarrassment and was often concealed from peers and adults. It was sometimes partial and caused only knee buckling, head and shoulder dropping, jaw sagging, and slurred speech. and was usually preceded by excessive daytime sleepiness. Sleep paralysis and hypnagogic hallucinations occurred in two thirds, at least once a week, and sometimes nightly. Polysomnograms showed sleep-onset REM sleep in 31. Multiple Sleep Latency Tests showed a mean sleep latency of $1.5 \mathrm{~min}+/-39$ secs; all children had 2 sleep-onset rapid eye movement sleep episodes in this test. HLA class II serotyping was positive for DQw6 in 46 children, and for DRw15 in 45. EEGs were "unremarkable," except for rapid onset of sleep. MRI in 14 and CT in 3 were normal. IQ mean score was $96+/-11$. Drug treatments included pemoline, methylphenidate, clomipramine, and fluoxetine; poor compliance was common, particularly after 7 years of age. Depressive reactions related to the illness, withdrawal from social interaction, poor selfesteem, and inadequate teacher understanding required psychological intervention. (Guilleminault C, Pelayo R. Narcolepsy in prepubertal children. Ann Neurol Jan 1998;43:135-142). (Respond: Dr Christian Guilleminault, Stanford Sleep Disorder Clinic, 401 Quarry Rd, Stanford, CA 94305).

COMMENT. Narcolepsy is uncommon during childhood, accounting for about $5 \%$ of cases attending a specialty clinic. The diagnosis is often delayed, and symptoms are mistaken for epilepsy, attention deficit disorder, learning and behavior problems. Early pharmacotherapy and counselling are recommended to prevent depressive reactions, school failures, and poor peer relationships. Delay in treatment results in irregular school attendance, academic failure, and poor self-esteem.

\section{NOOGENIC SEIZURES}

Self induced noogenic (thought provoked) absence seizures are reported in a photosensitive epileptic patient, a 20 year old man, treated at St Thomas' Hospital, London, UK. A febrile seizure at age 4 was followed by generalized tonic-clonic seizures precipitated by television at age 11 years and the onset of absence seizures at 12 years, coinciding with the time of his father's death. Photosensitivity to frequencies from 10 to $50 \mathrm{~Hz}$ were accompanied by $3-4 \mathrm{~Hz}$ polyspike and wave EEG discharges. Seizures were refractory to sodium valproate at age 15 , but were controlled by the addition of lamotrigine at age 18 and his introduction to university life. When seen at 20 years, he retrospectively admitted to having self induced seizures by thinking about his deceased father and the time he spent with him in the hospital. (Koutroumanidis M, Agathonikou A, Panayiotopoulos CP. Self induced noogenic seizures in a photosensitive patient. I Neurol Neurosurg \& Psychiatry Jan 1998;64:139-140). (Respond: Dr M Koutroumanidis, Department of Clinical Neurophysiology and Epilepsies, St Thomas' Hospital, Lambeth Palace Rd, London SE1 7EH, UK).

COMMENT. Reflex epilepsies can be precipitated by external stimuli, eg flickering lights or patterns, or by internal complex stimuli, eg thought processes. The latter are more difficult to diagnose, and are uncovered only by detailed history and patient admission. Emotional factors were obviously involved 